\title{
A single institution experience of total lymphoid irradiation using helical tomotherapy as part of the conditioning regimen of transplantation for severe aplastic anemia patients
}

\author{
Pei-Hsuan Lee ${ }^{1}$, Jian-Kuen Wu ${ }^{1,2}$, Miao-Ci Wang ${ }^{1}$, Sze-Hwei Lee ${ }^{3}$, Sung-Hsin Kuo ${ }^{1,4,5,6}$, Yu-Hsuan Chen ${ }^{1}$ \\ ${ }^{1}$ Division of Radiation Oncology, Department of Oncology, National Taiwan University Hospital, Taipei, Taiwan; ${ }^{2}$ Institute of Electro-Optical \\ Science and Technology, National Taiwan Normal University, Taipei, Taiwan; ${ }^{3}$ Tai Cheng Stem Cell Therapy Center, National Taiwan University \\ Cancer Center, Taipei, Taiwan; ${ }^{4}$ Graduate Institute of Oncology, ${ }^{5}$ Cancer Research Center, ${ }^{6}$ National Taiwan University Cancer Center, National \\ Taiwan University College of Medicine, Taipei, Taiwan \\ Contributions: (I) Conception and design: PH Lee, YH Chen; (II) Administrative support: SH Kuo; (III) Provision of study materials or patients: YH \\ Chen; (IV) Collection and assembly of data: PH Lee, JK Wu, MC Wang; (V) Data analysis and interpretation: PH Lee, SH Lee, SH Kuo, YH Chen; \\ (VI) Manuscript writing: All authors; (VII) Final approval of manuscript: All authors. \\ Correspondence to: Yu-Hsuan Chen. Division of Radiation Oncology, Department of Oncology, National Taiwan University Hospital, No. 1, Changde \\ St., Zhongzheng Dist., Taipei City 10048, Taiwan. Email: yhchen@ntuh.gov.tw.
}

Background: Total lymphoid irradiation (TLI) plays a role in the conditioning of hematopoietic stem cell transplantation (HSCT) from non-sibling donors for severe aplastic anemia (SAA) patients. For the past decades, an anterior-posterior opposed technique has been used to deliver TLI. We present our singleinstitution experience of helical tomotherapy TLI.

Methods: From October 2014 to February 2019, three consecutive patients of SAA who underwent tomotherapy TLI for pre-transplantation conditioning were enlisted. We retrospectively analyzed the survival, engraftment outcome, and toxicities of these patients and created a conventional TLI plan with anterior-posterior opposed fields for each patient to compare the dosimetry difference between helical tomotherapy and the conventional technique.

Results: Among all three patients, the engraftment rate was $100 \%$. The 18 -month overall survival and event-free survival were both $66.7 \%$ among the 3 patients ( 2 underwent unrelated donor transplantation and 1 underwent haplo-identical related donor transplantation), and $100 \%$ for the patients who received transplantation from matched- or mismatched-unrelated donors. No patient experienced acute grade 3 or worse graft-versus-host disease (GVHD), and none of the three patients suffered from late GVHD. No significant acute or late adverse events of radiation were observed. Tomotherapy-based TLI provides better target volume coverage with an average $10 \%$ higher coverage along with greater sparing of normal tissues. The total body volume exposed to 5 Gy was an average 35\% lower in the tomotherapy-based TLI.

Conclusions: TLI with non-ablative conditioning regimen provided excellent engraftment and an acceptable survival in the SAA patients who underwent non-sibling donor HSCT.

Keywords: Aplastic anemia (AA); helical tomotherapy; stem cell transplantation, hematopoietic

Received: 20 August 2019; Accepted: 02 March 2020; Published: 30 June 2020.

doi: $10.21037 /$ tro.2020.04.02

View this article at: http://dx.doi.org/10.21037/tro.2020.04.02 


\section{Introduction}

Aplastic anemia (AA) has rare incidence but it is 2- to 3 -time more common in Asia than in Western countries (1). AA is characterized by pancytopenia and a hypocellular bone marrow. Once the diagnosis is confirmed, the patient undergoes risk stratification for different treatment strategies. The diagnosis of severe aplastic anemia (SAA) has to meet the criteria of: (I) a bone marrow biopsy showing less than $25 \%$ to $30 \%$ of normal cellularity; or a bone marrow biopsy showing less than $50 \%$ normal cellularity in which fewer than $30 \%$ of the cells are hematopoietic, and (II) at least two of the following are present, absolute neutrophil count (ANC) $<500 / \mathrm{microL}$, platelet count $<20,000 / \mathrm{microL}$, or absolute reticulocyte count (ARC) $<60,000 /$ microL (2). A SAA patient with an identical HLA sibling donor could be cured by hematopoietic stem cell transplantation (HSCT). For the patients who lack identical HLA sibling donors, they could be treated with transplantation from unrelated donors if immunosuppressive drugs fail. Figure 1 summarizes the treatment protocol for SAA at our institution. The preparative conditioning regimen of HSCT from an unrelated donor may include total lymphoid irradiation (TLI). For the past decades, TLI was administered through a linear accelerator with anterior-posterior opposed fields, mainly the mental and the inverted-Y fields. This study aimed to retrospectively review the SAA patients who underwent TLI with tomotherapy as part of their transplantation conditioning in our institution.

\section{Methods}

\section{Patients}

We retrospectively reviewed registry data from our institute beginning from October 1, 2014; consecutively, 3 patients were enrolled in our study. Patients were eligible for the study if they had received a diagnosis of SAA and were treated with TLI as part of the conditioning regimen of HSCT with tomotherapy. Observations were carried out through February 1, 2019. The diagnosis of SAA was based on decreased cellular counts in at least 2 cell lines in the peripheral blood, and a severe hypocellular marrow with cellularity less than $25 \%$. This study was approved by the National Taiwan University Hospital Research Ethics Committee Office (approval ID: 202001040RIN).

\section{Treatment}

All of three patients received TLI, antithymocyte globulin, and cyclophosphamide as the non-myeloablative conditioning regimen prior to HSCT. Treatment protocol for SAA patients in National Taiwan University Children's Hospital is illustrated in Figure 2. TLI was administered from a 6-MV tomotherapy (photon beam) at a dose of 200 cGy daily, starting 10 days before transplantation, until a total of 4 doses ( $800 \mathrm{cGy}$ ) had been delivered. The clinical target volumes (CTVs) included the cervical, axillary, mediastinal, para-aortic, pelvic and inguinal nodal regions, and the spleen. The planning target volumes (PTVs) were CTVs plus a $5-10 \mathrm{~mm}$ margin to compromise the internal organ motions and set-up errors. For this study, we used the $25-\mathrm{mm}$ slice thickness, and a pitch of 0.287 was used in the planning of the patients. In the 3 rd patient, a hemisphere lead block was used for testis protection due to the short distance between the gonads and the target volume.

These 3 patients received cyclophosphamide at a dose of $50 \mathrm{mg}$ per $\mathrm{kg}$ of body weight per day on day-5 through -2 , with day 0 being the day of transplantation. Rabbit antithymocyte globulin, at a total dose of 6-9 $\mathrm{mg}$ per $\mathrm{kg}$ of body weight, was given intravenously on day-4 through -2 . Cyclosporine A (CsA) and a short-course methotrexate were used as GVHD prophylaxis.

\section{Dosimetry comparison}

For every single patient, we created a conventional 2D plan of TLI for each patient, based on the identical CT image and the same target volumes used for the tomotherapy planning. The conventional plans were used merely for dosimetry reviewing, not for the actual treatment. The conventional TLI includes two compartments, the mantle field and the inverted-Y field. Both fields were built by lead blocks. To fairly evaluate the dose-volume distribution of these 2 different techniques, the plans were based on the identical CT image and identical contouring for CTV, PTV, and organs of risk, for each patient. For all three conventional 2D plans, we use the Pinnacle treatment planning system for dose calculation, with $10 \mathrm{MV}$ photons, and assuming the transmission factor as 0.96 for the blocks.

\section{Results}

\section{Patients' characteristics}

Table 1 summarizes the clinical features of the 3 patients enrolled in the study. All of them were classified as SAA according to the aforementioned criteria. There were no 


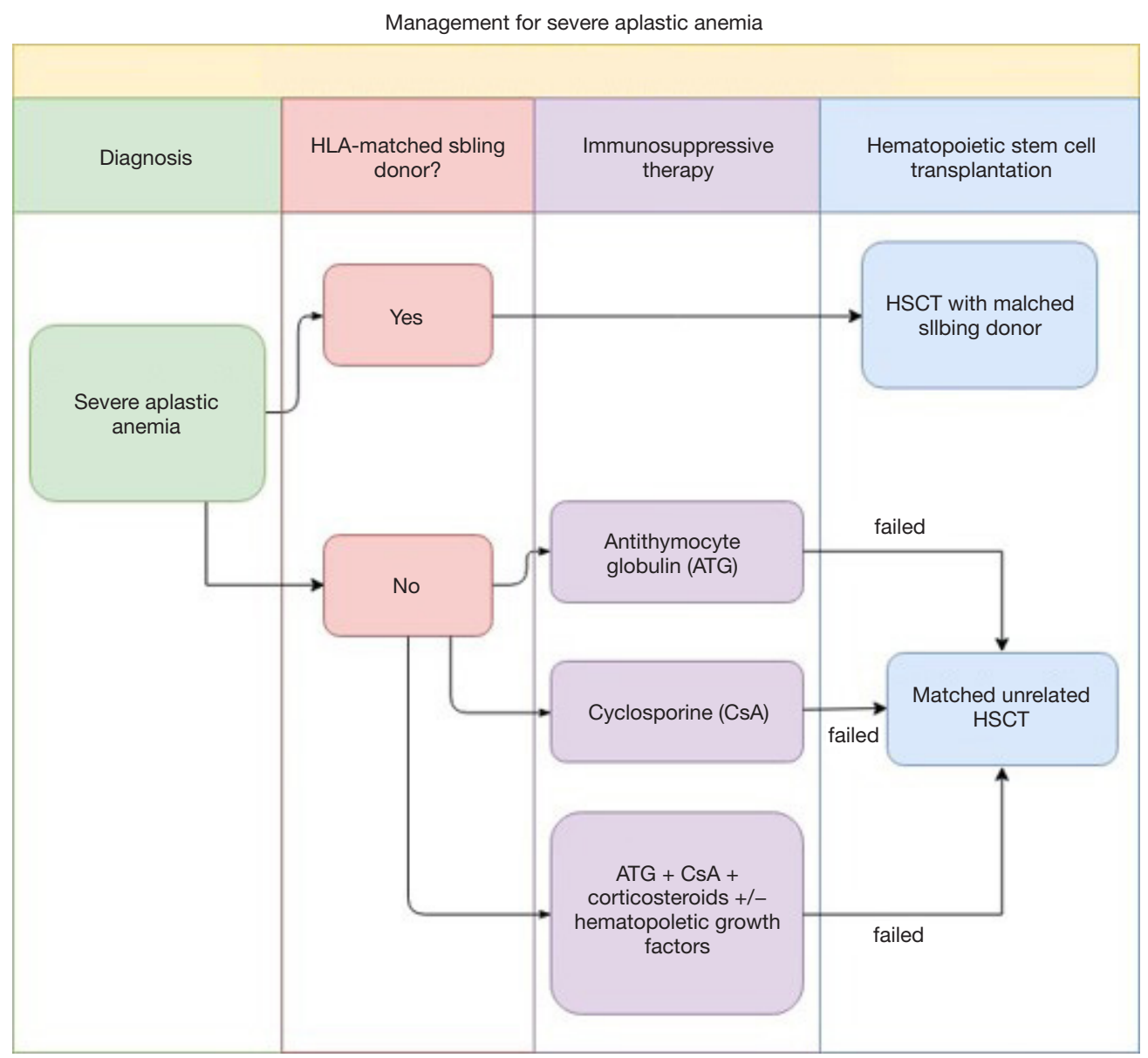

Figure 1 The management protocol for severe aplastic anemia for children and young adults at NTUCH. NTUCH, National Taiwan University Children's Hospital; HSCT, hematopoietic stem cell transplantation.

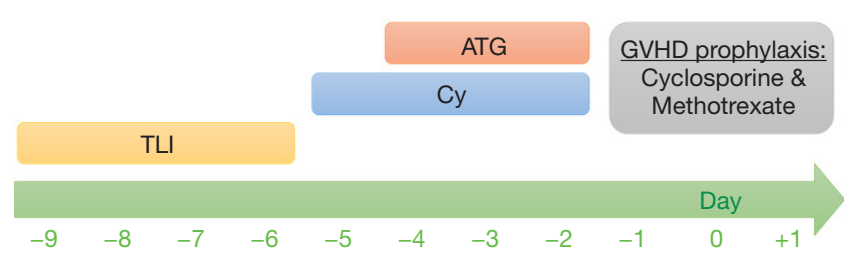

Figure 2 The protocol for transplantation of SAA patients at NTUCH. NTUCH, National Taiwan University Children's Hospital; TLI, total lymphoid irradiation; Cy, Cyclophosphamide; ATG, anti-thymocyte globulin; SAA, severe aplastic anemia.

records of congenital abnormality, mental retardation, or family history of hematological disorder in the 3 patients. Every patient was first treated with steroid, and 1 to 2 courses of immunosuppressive therapy (thymocyte globulin + Cyclosporine A). They underwent HSCT from being refractory to immunosuppressive therapy. The age at the time of transplantation were 11,21 , and 8 , respectively. The first and the third patients required peripheral blood stem cell transplants of matched or mismatched unrelated donors from the Buddhist Tzu Chi Stem Cell Center (BTCSCC) because they did not otherwise have an HLA-identical sibling donor. Although bone marrow transplantation holds lower risk of acute and chronic GVHD than peripheral-blood stem cell transplantation (3), it was not easy to find an unrelated donor who was willing to harvest bone marrow stem cells from the pelvic bone. The second patient, the oldest one, without a matched unrelated donor, underwent haplo-identical bone marrow transplant from the patient's father. She was transfused with a non-T-cell-depleted product with CD34+ $1.59 \times 10^{\wedge^{6}}$ and CD3 $2.39 \times 10^{\wedge}{ }^{6}$ cells $/ \mathrm{kg}$. Post-transplant cyclophosphamide (PT-Cy) has gained popularity in recent years due to the 
Table 1 The patient characteristics

\begin{tabular}{|c|c|c|c|}
\hline Characteristics & The 1st patient & The 2nd patient & The 3rd patient \\
\hline Gender & Male & Female & Male \\
\hline Previous treatment & ATG + CsA + steroid (II) & Steroid; ATG + CsA (I) 2009/7/29 & Steroid; ATG + CsA (I) \\
\hline Initial peripheral cell count & 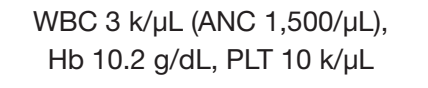 & $\begin{array}{c}\text { WBC } 3.25 \mathrm{k} / \mu \mathrm{L} \text { (ANC } 1.1 \mathrm{k} / \mu \mathrm{L}) \\
\mathrm{Hb} 7.8 \mathrm{~g} / \mathrm{dL}, \text { PLT } 21 \mathrm{k} / \mu \mathrm{L}\end{array}$ & $\begin{array}{c}\text { WBC } 1.21 \mathrm{k} / \mu \mathrm{L} \text { (ANC } 72.6 / \mu \mathrm{L}), \\
\mathrm{Hb} 6.2 \mathrm{~g} / \mathrm{dL}, \text { PLT } 2 \mathrm{k} / \mu \mathrm{L}\end{array}$ \\
\hline Donor & Unrelated, HLA matched (10/10) & $\begin{array}{l}\text { Related (father), Haplo-identical } \\
\qquad \operatorname{HLA}(5 / 10)\end{array}$ & $\begin{array}{l}\text { Unrelated, HLA mismatched } \\
\qquad(9 / 10)\end{array}$ \\
\hline Conditioning regimen & $\begin{array}{c}\text { TLI (8 Gy in } 4 \text { fractions) + } \\
\text { Endoxan + ATG }\end{array}$ & $\begin{array}{c}\text { TLI (8 Gy in } 4 \text { fractions) + } \\
\text { Endoxan + ATG }\end{array}$ & $\begin{array}{c}\text { TLI (8 Gy in } 4 \text { fractions) + } \\
\text { Endoxan + ATG }\end{array}$ \\
\hline \multicolumn{4}{|l|}{ Pretransplant CMV status } \\
\hline Recipient & $\begin{array}{l}\lg \mathrm{M}(-), \lg \mathrm{g}(+), \text { viral load: } \\
\text { undetectable }\end{array}$ & $\begin{array}{l}\operatorname{lgM}(-), \lg G(+), \text { viral load: } \\
\text { undetectable }\end{array}$ & $\begin{array}{l}\operatorname{lgM}(-), \lg G(+), \text { viral load: } \\
\text { undetectable }\end{array}$ \\
\hline Donor & $\lg M(-), \lg G(+)$ & $\lg M(-), \lg G(+)$ & $\lg M(-), \lg G(+)$ \\
\hline
\end{tabular}

ATG, anti-thymocyte globulin; CsA, cyclosporine A; ANC, absolute neutrophil count; ARC, absolute reticulocyte count; MUD-PBSCT, matched unrelated donor peripheral blood stem cell transplantation; MMUD, mismatched unrelated donor; BMT, bone marrow transplantation; TLI, total lymphoid irradiation; CMV, cytomegalovirus; HLA typing, A, B, C, DR, DQ.

potential to reduce GVHD for allogenic transplantation with an acceptable safety profile (4). However, none of our patients received post-transplant cyclophosphamide. The transplantation protocol of our hospital for children SAA at that time did not include the use of PT-Cy.

\section{Engraftment}

Engraftment after HSCT is defined as an ANC greater than $0.5 \times 10^{9} / \mathrm{L}$ on the first day of three consecutive days (5). Engraftment of these 3 patients occurred on the posttransplant days 17, 11, and 12, respectively. At 1-month post-transplantation, two of the patients' peripheral blood genotypes were identical to that of the donors on short tandem repeat (STR) genotype analysis. Primary graft failure is characterized by the absence of initial donor cell engraftment (donor cells less than $95 \%$ ) or peripheral blood ANC $<0.5 \times 10^{9} / \mathrm{L}$ by day +28 after allo-HSCT from peripheral blood or bone marrow progenitors in the absence of relapse. The engraftment rate was $100 \%$, with no cases of primary graft failure (Table 1). Engraftment was seen after a median of 12 days (range, 11-17 days). However, the second patient experienced fatal infection complication of cytomegalovirus (CMV) viremia two and a half months after bone marrow transplantation. This patient eventually died from sepsis 3 months after transplantation. HSCT successfully transformed the two survived patients from transfusion-dependent to transfusion-independent status.

\section{Patient survival}

The first and the third patient received hematopoietic stem cell from matched or mismatched unrelated donors; they were still alive and under regular clinical follow-up when this analysis closed. However, the second patient died from CMV viremia 3 months after transplantation. She had been treated with ganciclovir after a plasma CMV viral load of $24,000 \mathrm{cp} / \mathrm{mL}$ was found on day +49 . In combination with ganciclovir, CMV intravenous immunoglobulins were added on day +57 . The antiviral treatment was shifted to Foscarnet due to her pancytopenia and CMV viremia progression on day +62 (with a CMV viral load of 202,000 cp/mL). Despite our treatment, she eventually died from sepsis on day +95 . Overall survival is defined as the time period from day 0 of transplantation to death. Event-free survival is defined as the time period from day 0 of transplantation till any of the following events occurs: the recurrence of SAA, secondary graft failure, or death. The 18-month overall survival and 
Table 2 The acute adverse events related to transplantation

\begin{tabular}{llll}
\hline Adverse events & The 1st patient & The 2nd patient & The 3rd patient \\
\hline $\begin{array}{l}\text { Chemotherapy or immunosuppressant } \\
\text { related toxicity }\end{array}$ & CsA related AKI & $\begin{array}{l}\text { Septic shock (CMV viremia), Post-transplant lymphoproliferative disorder, Gr. } \\
\text { acute kidney injury }\end{array}$ & $\begin{array}{l}4 \text { neutropenia, Gr. 2 nausea, and Gr. 1 vomiting } \\
\text { Radiation related toxicity }\end{array}$ \\
$\begin{array}{llll}\text { Gr. 1 nausea and } \\
\text { vomiting }\end{array}$ & Absent & Absent \\
GVHD, acute & Stage 1 Gl, acute & Stage 2 skin & Stage 2-3 skin \\
GVHD, chronic & Absent & Absent & Absent
\end{tabular}

CsA, cyclosporine A; AKI, acute kidney injury; CMV, cytomegalovirus.

event-free survival were both $66.7 \%$ among the 3 patients, and were $100 \%$, if the second patient who underwent haplo-identical transplantation was excluded. The median survival was not reached.

\section{Adverse events and graft-versus-bost disease (GVHD)}

Standard scores for GVHD on a scale from grade 0 through grade 4 were used during the first 100 days after transplantation to diagnose acute GVHD (6), and thereafter, patients were evaluated for chronic GVHD, which was classified as absent, limited, or extensive (7).

Of the three patients, non-demonstrated grade 2 or worse radiation adverse events occurred. Table 2 listed the adverse events of these 3 patients. Aside from the side effects caused by the regimens used in transplantation, all of the patients experienced acute GVHD of variable severity. The first patient experienced stage 1 gastrointestinal tract involvement; the second patient suffered from stage 3 skin involvement; the third patient demonstrated stage 2 skin erythema. No one experienced acute grade 3 or worse GVHD. Although the GVHD were well-controlled by cyclosporine and methotrexate, the first patient eventually developed cyclosporine-related acute renal injury. None of these patients experienced chronic GVHD.

\section{Dose-volume distribution}

Table 3 shows the dose-volume distribution characteristics using tomotherapy and the dose-volume differences from tomotherapy-based plans to conventional 2D plans. Tomotherapy provided a better treatment target coverage with a median absolute increase by $9.76 \%$ of PTV coverage (an average $10 \%$ higher than $2 \mathrm{D}$ plans), while reducing the global maximum dose. Tomotherapy yielded better homogeneity of dose distribution and omitted the dose uncertainty caused by the field junctions of the mantle and the inverted $\mathrm{Y}$ fields. As the organs of interest, tomotherapy demonstrated reduced or similar maximal point dose. Furthermore, it reduced the volume of the medium to low dose exposure. The decreased percentage volume of 5 Gy (V5) of tomotherapy-based plans reflected this effect, especially for the organs near the treatment targets, like lung, heart, liver, stomach, kidneys, and skin. There was a median absolute reduction of $17.19 \%$ in the volume of the total body exposed to 5 Gy or more (V5) by using tomotherapy (an average $35 \%$ lower than $2 \mathrm{D}$ plans) (Table 3 and Figure 3), which perhaps could transfer to lower secondary malignancy rate in the future. Figure 3 illustrates the isodose distribution of tomotherapy-based and 2D plans respectively. Tomotherapy not only reduced the high dose area (such as the volume of $\geq 105 \%$ prescribed dose) but also eliminated the uncertain dose distribution caused by field junctions.

\section{Discussion}

The definitive treatment modalities of acquired SAA are immunosuppression or hematopoietic stem-cell transplantation. Therapy involves combination of ATG and cyclosporine to form intensive immunosuppression for SAA patients. For patients that had a matched sibling donor, a full recovery is achievable by hematopoietic stemcell transplantation, from an allogenic HLA-matched donor, and with excellent long-term results. With the aid of improvement in conditioning regimens and GVHD prophylaxis, the most encouraging survival achieved $80 \%$ to $90 \%$ at 5 years or longer (8-12). However, only $20 \%$ to $30 \%$ of patients have available matched sibling donors. Thus, unrelated donors become an alternative source of HSCT.

Unrelated donor transplant is currently offered for children without matched sibling donors who have 
Table 3 The dose-volume parameters and the difference between tomotherapy and $2 \mathrm{D}$ plans

\begin{tabular}{|c|c|c|c|}
\hline \multirow[b]{2}{*}{ Targets } & \multicolumn{2}{|c|}{ The median value (Gy or \%) } & \multirow{2}{*}{$\begin{array}{l}\text { Average dose } \\
\text { increase/ } \\
\text { reduction (\%) }\end{array}$} \\
\hline & Tomotherapy & $\begin{array}{c}\text { Conventional } \\
\text { 2D }\end{array}$ & \\
\hline CTV & 99.9 & 94.04 & 8.14 \\
\hline PTV & 96.33 & 88.17 & 10.01 \\
\hline Global maximum & 109.63 & 116.13 & -5.6 \\
\hline Brain stem max & 1.68 & 1.47 & 44.33 \\
\hline Spinal cord max & 6.72 & 8.6 & -29.21 \\
\hline Cauda equine max & 7.58 & 8.68 & -10.3 \\
\hline Eye L. max & 0.48 & 2.84 & -40.67 \\
\hline Eye R. max & 0.31 & 2.73 & -38.97 \\
\hline Lens L. max & 0.2 & 2.23 & -66.58 \\
\hline Lens R. max & 0.19 & 2.19 & -65.61 \\
\hline Optic nerve L. max & 0.24 & 1.45 & -60.46 \\
\hline Optic nerve R. max & 0.24 & 1.5 & -65.17 \\
\hline Parotid L. max & 8.37 & 8.69 & -2.91 \\
\hline Parotid R. max & 8.44 & 8.58 & -2.09 \\
\hline Ear L. max & 1.04 & 0.55 & 83.85 \\
\hline Ear R. max & 0.45 & 0.61 & 70.24 \\
\hline Lung max & 8.53 & 8.79 & -2.61 \\
\hline Mean & 5.08 & 4.62 & 4.87 \\
\hline V5 & 49.85 & 48.93 & -5.48 \\
\hline Heart max & 8.51 & 8.61 & -1.05 \\
\hline Mean & 6.18 & 6.77 & -12.28 \\
\hline V5 & 60.71 & 81.86 & -31.09 \\
\hline Liver max & 8.38 & 8.63 & -2.58 \\
\hline Mean & 3.25 & 2.57 & 16.77 \\
\hline V5 & 19.76 & 27.24 & -46.76 \\
\hline Stomach max & 8.46 & 8.68 & -3.07 \\
\hline V5 & 64.16 & 95.13 & -32.83 \\
\hline Kidney L. max & 8.46 & 8.76 & -3.28 \\
\hline V5 & 43.41 & 78.8 & -35.09 \\
\hline Kidney R. max & 7.34 & 8.18 & -8.54 \\
\hline V5 & 1.47 & 16.61 & -71.12 \\
\hline Bowl max & 8.57 & 8.8 & -1.77 \\
\hline V5 & 26.94 & 47.53 & -40.11 \\
\hline
\end{tabular}

Table 3 (continued)
Table 3 (continued)

\begin{tabular}{|c|c|c|c|}
\hline \multirow[b]{2}{*}{ Targets } & \multicolumn{2}{|c|}{ The median value (Gy or \%) } & \multirow{2}{*}{$\begin{array}{l}\text { Average dose } \\
\text { increase/ } \\
\text { reduction (\%) }\end{array}$} \\
\hline & Tomotherapy & $\begin{array}{c}\text { Conventional } \\
2 \mathrm{D}\end{array}$ & \\
\hline Bladder max & 8.57 & 8.45 & 59.24 \\
\hline V5 & 20.28 & 58.46 & -31.23 \\
\hline Rectum max & 7.38 & 8.37 & -4.34 \\
\hline V5 & 11.1 & 30.78 & -1.47 \\
\hline Testis/penis max & $<1$ & 4.93 & $-82.35^{\dagger}$ \\
\hline Ovaries max & Scattered & 0.13 & $-^{\dagger}$ \\
\hline Skin $\max ^{*}$ & 8.58 & 9.22 & -5.4 \\
\hline V5 ${ }^{\star}$ & 7.45 & 22.49 & -79.12 \\
\hline Whole body max & 8.77 & 9.29 & -5.48 \\
\hline V5 & 20.29 & 37.15 & -34.96 \\
\hline $\begin{array}{l}\text { Femoral head L. } \\
\max \end{array}$ & 8.11 & 8.29 & -1.14 \\
\hline $\begin{array}{l}\text { Femoral head } \mathrm{R} \text {. } \\
\text { max }\end{array}$ & 7.18 & 8.35 & -9.33 \\
\hline
\end{tabular}

Since the brain stem, eyes, lens, optic nerves, ears, bladder, rectum, and femoral heads of the second patient only have scattered dose or almost 0 , it's hard to evaluate the differences between the tomotherapy and 2D plans. The table only showed the values of the average of the first and third. *, only the $3^{\text {rd }}$ patient had dose-volume data of "skin" (the area from cutaneous surface to $0.5 \mathrm{~cm}$ depth of subcutaneous); the other two patients use "whole body" as substitution for evaluation; ${ }^{\dagger}$, the data represents the dose-volume difference of the 3rd patient, since the "testis and penis" of the 1st patient and the "ovaries" of the 2nd patient received only scattered dose in both tomotherapy-based and 2D plans due to the distance from target volumes.

very severe disease or have failed one or two courses of immunosuppressive therapy, and adults who have failed multiple courses immunosuppression therapy and or alternative therapies such as androgens (13). Unlike allogeneic sibling transplants, transplants from unrelated donors may require irradiation to ensure engraftment, perhaps, due to the source of the donor cells and the transfusion status of the recipient. In a multicenter study at Seattle, 62 SAA patients underwent matched unrelated stem-cell transplantation, with preparative conditioning regimen of cyclophosphamide, ATG and total body irradiation (TBI); the graft failure rate was $2 \%$, grade II to IV acute GVHD was observed in 70\%, chronic GVHD 

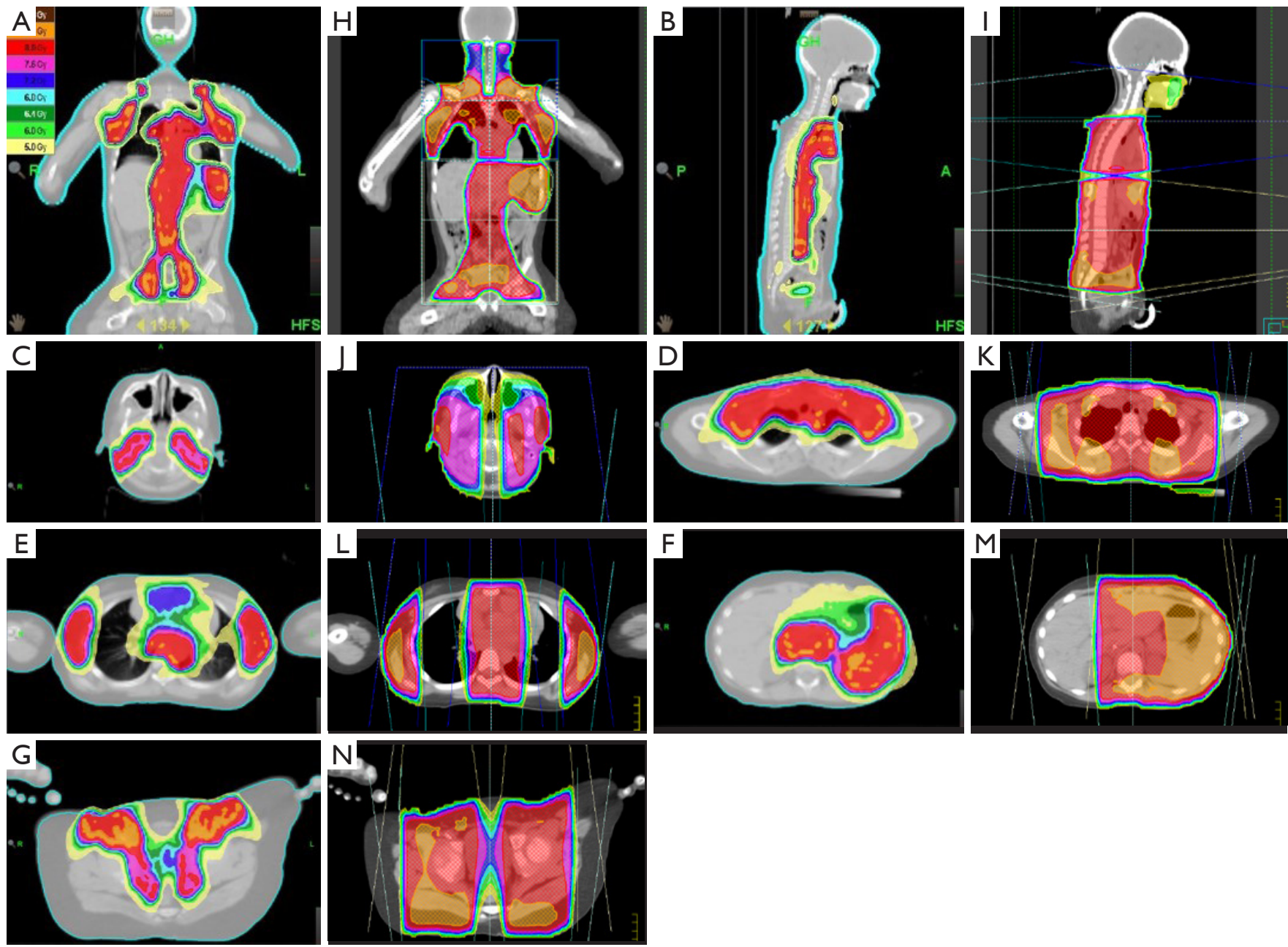

Figure 3 The isodose curves. The isodose curves of tomotherapy (A,B,C,D,E,F) and 2D (G,H,I,J,K,L,M,N) plans. The tomotherapy-based plan has more homogenous dose distribution. The anterior-posterior opposed technique generates more high-dose area and the under-dose area at field junction.

was observed in $52 \%$, and overall survival was $61 \%$ at 5 years (14). On the other hand, 25 patients who had HLA-nonidentical stem cell transplants in the same study, sustained engraftment was achieved in $88 \%$ and 5 -year overall survival was $44 \%$. In contrast, a European registry substituted irradiation with fludarabine for unrelated and mismatched family donors (15). Eighteen percent of the recipients had graft failure. In prospective studies of alternative donor stem-cell transplantation for SAA with irradiation constituting the conditioning regimen, the overall survival ranges from $56 \%$ to $100 \%(14,16,17)$.

Conditioning with TLI plus antithymocyte globulin provides a protective effect against the development of acute GVHD after hematopoietic-cell transplantation (18).
In this study, thirty-seven patients with lymphoid malignant diseases or acute leukemia underwent hematopoietic-cell transplantation from HLA-matched related or unrelated donors using TLI plus antithymocyte globulin as the conditioning regimen. Only 2 of the 37 patients had acute GVHD. The study also observed an increased production of interleukin-4 and a reduced proliferative response of donor CD4+ $\mathrm{T}$ cells from patients who underwent conditioning with TLI, rather than TBI, which was mandatory for reducing the incidence of acute GVHD.

Lee et al. retrospectively evaluated twenty SAA patients who were multi-transfused or who had graft failure from prior HSCT (19). The conditioning regimen prior to allogeneic HSCT was ATG plus a single fraction TLI with 
a median dose of 7.5 Gy in 1 fraction. Two primary graft failures and 4 late graft failures were found later. With a median follow-up of 10.8 years, the overall survival rate was $83.1 \%$ at 10 years. The acute and chronic GVHD rates were both $20 \%$. The authors chose single-fraction TLI because they wanted to shorten the radiation treatment period for these immunosuppressed and pancytopenic patients. Unlike patients with hematological malignancies, marrow aplasia disorders do not require large irradiation fields. The limited fields would lower the incidence of pneumonitis, as well as the impact on growth, endocrine, or fertility of children or young patients. To our knowledge, currently, there is no randomized trial comparing singleor multi-fraction TLI. One landmark trial randomized 53 non-lymphoblastic leukemia patients in their first complete remission to $10 \mathrm{~Gy}$ in a single exposure, or $2 \mathrm{~Gy} /$ day for 6 days TBI before an allogeneic marrow transplant (20). The fractionated arm had a significant overall survival benefit over the single-fraction arm. Fewer patients in the fractionated arm developed interstitial pneumonitis and severe acute GVHD. Based on the experience with TBI, our institute chose multiple fraction TLI rather than a single exposure.

Each of the 3 patients in our study represented the recipient of HLA-matched unrelated donor, haploidentical related donor, and HLA-mismatched unrelated donor, respectively. The engraftment rate was $100 \%$. Despite engraftment, the recipient of haploidentical transplantation died of infectious complications on day +95 . After a median follow-up of 563 days, the overall survival rate was $66.7 \%$ for the whole cohort, which was comparable with the historical data. Older age and haploidentical transplantation from a family member are both poor prognostic factors for transplantation (21). This could partially explain the early death of the second patient. For transplantation from HLA-matched and HLA-mismatched (9/10) unrelated donors, the overall survival was $100 \%$ at 18 months in our study. None of the 3 patients experienced grade 3 or worse GVHD. This could again confirm that TLI has an effect of decreasing GVHD.

TLI has been traditionally administered through a linear accelerator or a cobalt-60 machine with anterior-posterior opposed fields toward the major lymph node regions and spleen. Bony landmarks work as reference points for patient setup. Due to the limited field length of a linear accelerator and a cobalt-60 machine, this technique requires changing patient's position, which may result in dose heterogeneity. The treatment field length of tomotherapy may expand to up to $130 \mathrm{~cm}$ through the translational movement of the couch. Helical tomotherapy combines image-modulated radiotherapy with megavolt computed tomographic image guidance. Tomotherapy technique provides not only improvement of dose homogeneity, precise treatment positioning, but also satisfying coverage of the nodal and splenic CTVs with a rapid drop-off of dose near the critical structures.

McCutchen et al. reported a single institution's experience of TLI performed by helical tomotherapy in an adult patient with late-onset cardiac transplant rejection (22). The prescribed dose was 8 Gy to $95 \%$ of PTV, in 10 twiceweekly fractions. Tomotherapy intensity-modulated TLI relieved the rejection episode and allowed minimization of dose to critical organs, especially the spinal cord, kidneys, liver, and lungs. Within the first month of post-radiation follow-up, the patient was well-tolerated without recorded acute adverse event. Balasz et al. compared the dose distributions of TLI from 4 irradiation techniques, with a dose of 12 Gy in 3 fractions: IMRT, VMAT, tomotherapy to conventional radiation (23). Tomotherapy obtained the highest minimum dose and V95\% (volume covered by $95 \%$ prescribed dose) for CTV. The lenses, spinal cord, heart, and parotid glands benefited most from dose reduction in relation to the conventional plan by using rotational techniques. The conventional technique performed superiorly on sparing lungs (with lower mean and median doses), liver, and right kidney.

Since TLI covers a large part of body volume, secondary malignancy should also be taken under consideration. The direct correlation with secondary malignancy to TBI is difficult to distinguish due to the general use of immunosuppressive medication in such a population. The most commonly mentioned secondary malignancies seen following TLI series are lymphomas, along with skin cancers, with the rate of $2-12 \%$ (24-26). Hall et al. suggested that the dose-response relationship for radiationinduced cancer is complex: the risk increases with doses up to about $4 \mathrm{~Gy}$, and they strongly favor a model with a plateau after a dose of about 5 Gy (27). Thus, to reduce the volume exposed to a dose of $5 \mathrm{~Gy}$ or more as low as possible seems reasonable. To the best of our knowledge, this is the first study to directly compare the dosimetry characteristics of tomotherapy and anterior-posterior opposed technique TLI in SAA patients with available engraftment and survival outcome results. In our study, tomotherapy apparently reduced the volume of low dose exposure compared to the conventional anterior-posterior opposed technique. 


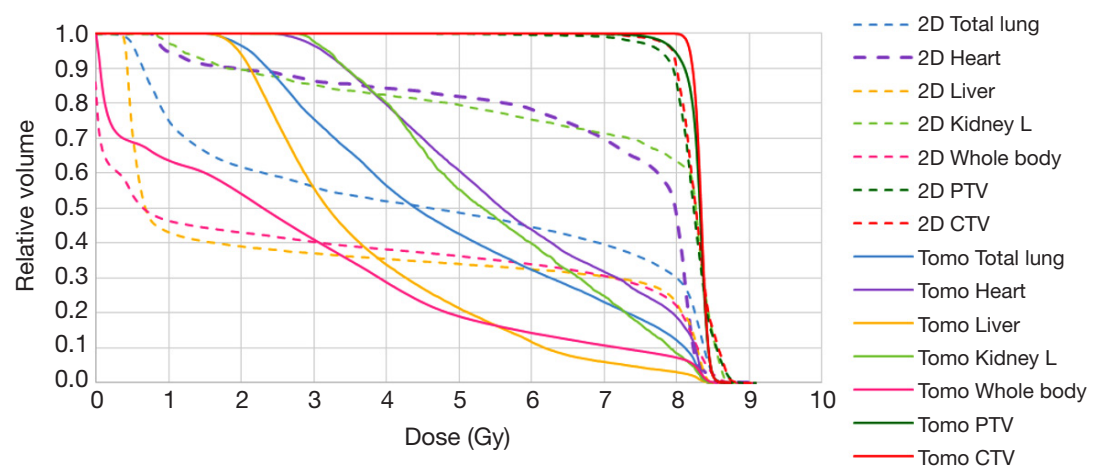

Figure 4 The DVH comparison. The dose-volume histogram of tomotherapy-based (solid lines), and anterior-posterior opposed 2D (dashed lines) plans of the $3 \mathrm{rd}$ patient. The DVH curves of $2 \mathrm{D}$ plan shifts to the right, which implies that more parts of the body and OARs were under exposure of $\geq 5$ Gy. DVH, dose-volume histogram; OARs, organs at risk.

The whole body V5 decreased by an absolute $17.19 \%$, which indicates that tomotherapy had an average $35 \%$ lower V5 than 2D plans. Figure 4 shows the dose-volume histogram (DVH) for the total body V5 of tomotherapy and $2 \mathrm{D}$ technique respectively. This may probably result in a reduction of secondary malignancy risk. Apart from conventional 2D technique, intensity-modulated radiation therapy (IMRT) and volumetric arc therapy (VMAT) could achieve good coverage, but none of these techniques could avoid the uncertain dose distribution at the field junctions in TLI.

To conclude, we suggest tomotherapy as an ideal technique to deliver TLI.

There are still some limitations to our study. First, we only recruited 3 patients, a small representative sample. However, owing to the rare incidence of SAA and even rarer use of TLI in SAA transplantation, like in previous studies, we could only gather a small sample size. Thus, the study still holds value from the information collected from these rare disease states. Secondly, due to the retrospective feature of our study, we did not have an identical dose constraint criterion for each patient as is preferred. Thirdly, to define chronic events like GVHD or radiation-induced second malignancy, as well as the survival outcome, we need a longterm follow-up over several years to identify effectiveness of treatments.

\section{Conclusions}

From our single-institution experience, tomotherapy-based TLI plus non-ablative regimens for pre-transplantation conditioning achieves an excellent engraftment rate (100\%) as well as a satisfying survival in SAA patients undergoing hematopoietic stem cell transplant with non-sibling donors. TLI ensured the engraftment and reduced the occurrence of GVHD. Besides, tomotherapy-based TLI takes advantage of lessening the volume of low dose exposure and avoiding the field junctions. Perhaps this benefit may further transfer to lower secondary malignancy rate in a long-term follow-up.

\section{Acknowledgments}

Funding: None.

\section{Footnote}

Conflicts of Interest: All authors have completed the ICMJE uniform disclosure form (available at http://dx.doi. org/10.21037/tro.2020.04.02). SHK serves as an Associate Editors-in-Chief of Therapeutic Radiology and Oncology from Apr 2020 to Mar 2022. The other authors have no conflicts of interest to declare.

Ethical Statement: The authors are accountable for all aspects of the work in ensuring that questions related to the accuracy or integrity of any part of the work are appropriately investigated and resolved. The study was conducted in accordance with the Declaration of Helsinki (as revised in 2013). This study was approved by National Taiwan University Hospital Research Ethics Committee Office (approval ID: 202001040RIN). Informed consent was waived due to the retrospective nature of the study.

Open Access Statement: This is an Open Access article 
distributed in accordance with the Creative Commons Attribution-NonCommercial-NoDerivs 4.0 International License (CC BY-NC-ND 4.0), which permits the noncommercial replication and distribution of the article with the strict proviso that no changes or edits are made and the original work is properly cited (including links to both the formal publication through the relevant DOI and the license). See: https://creativecommons.org/licenses/by-nc-nd/4.0/.

\section{References}

1. Issaragrisil S, Kaufman DW, Anderson T, et al. The epidemiology of aplastic anemia in Thailand. Blood 2006;107:1299-307.

2. Rozman C, Marín P, Nomdedeu B, et al. Criteria for severe aplastic anaemia. Lancet 1987;2:955-7.

3. Anasetti C, Logan BR, Lee SJ, et al. Peripheral-blood stem cells versus bone marrow from unrelated donors. $\mathrm{N}$ Engl J Med 2012;367:1487-96.

4. Rashidi A, Slade M, DiPersio JF, et al. Post-transplant high-dose cyclophosphamide after HLA-matched vs haploidentical hematopoietic cell transplantation for AML. Bone Marrow Transplant 2016;51:1561-4.

5. Ozdemir ZN, Civriz Bozdağ S. Graft failure after allogeneic hematopoietic stem cell transplantation. Transfus Apher Sci 2018;57:163-7.

6. Glucksberg H, Storb R, Fefer A, et al. Clinical manifestations of graft-versus-host disease in human recipients of marrow from HL-A-matched sibling donors. Transplantation 1974;18:295-304.

7. Sullivan KM, Agura E, Anasetti C, et al. Chronic graftversus-host disease and other late complications of bone marrow transplantation. Semin Hematol 1991;28:250-9.

8. Gupta V, Ball SE, Yi QL, et al. Favorable effect on acute and chronic graft-versus-host disease with cyclophosphamide and in vivo anti-CD52 monoclonal antibodies for marrow transplantation from HLA-identical sibling donors for acquired aplastic anemia. Biol Blood Marrow Transplant 2004;10:867-76.

9. Kahl C, Leisenring W, Deeg HJ, et al. Cyclophosphamide and antithymocyte globulin as a conditioning regimen for allogeneic marrow transplantation in patients with aplastic anaemia: a long-term follow-up. Br J Haematol 2005;130:747-51.

10. Locatelli F, Bruno B, Zecca M, et al. Cyclosporin A and short-term methotrexate versus cyclosporin $\mathrm{A}$ as graft versus host disease prophylaxis in patients with severe aplastic anemia given allogeneic bone marrow transplantation from an HLA-identical sibling: results of a GITMO/EBMT randomized trial. Blood 2000;96:1690-7.

11. Camitta BM, Thomas ED, Nathan DG, et al. Severe aplastic anemia: a prospective study of the effect of early marrow transplantation on acute mortality. Blood 1976;48:63-70.

12. Passweg JR, Socié G, Hinterberger W, et al. Bone marrow transplantation for severe aplastic anemia: has outcome improved? Blood 1997;90:858-64.

13. Bacigalupo A. How I treat acquired aplastic anemia. Blood 2017;129:1428-36.

14. Deeg HJ, O'Donnell M, Tolar J, et al. Optimization of conditioning for marrow transplantation from unrelated donors for patients with aplastic anemia after failure of immunosuppressive therapy. Blood 2006;108:1485-91.

15. Bacigalupo A, Socie' G, Lanino E, et al. Fludarabine, cyclophosphamide, antithymocyte globulin, with or without low dose total body irradiation, for alternative donor transplants, in acquired severe aplastic anemia: a retrospective study from the EBMT-SAA Working Party. Haematologica 2010;95:976-82.

16. Kojima S, Inaba J, Yoshimi A, et al. Unrelated donor marrow transplantation in children with severe aplastic anaemia using cyclophosphamide, anti-thymocyte globulin and total body irradiation. Br J Haematol 2001;114:706-11.

17. Kojima S, Matsuyama T, Kato S, et al. Outcome of 154 patients with severe aplastic anemia who received transplants from unrelated donors: the Japan Marrow Donor Program. Blood 2002;100:799-803.

18. Lowsky R, Takahashi T, Liu YP, et al. Protective conditioning for acute graft-versus-host disease. $\mathrm{N} \mathrm{Engl} \mathrm{J}$ Med 2005;353:1321-31.

19. Lee YH, Kim JY, Choi BO, et al. Total lymphoid irradiation based conditioning for hematopoietic stem cell transplantation in severe aplastic anemia. Radiat Oncol J 2012;30:165-72.

20. Thomas ED, Clift RA, Hersman J, et al. Marrow transplantation for acute nonlymphoblastic leukemic in first remission using fractionated or single-dose irradiation. Int J Radiat Oncol Biol Phys 1982;8:817-21.

21. Woodard P, Cunningham JM, Benaim E, et al. Effective donor lymphohematopoietic reconstitution after haploidentical CD34+-selected hematopoietic stem cell transplantation in children with refractory severe aplastic anemia. Bone Marrow Transplant 2004;33:411-8.

22. McCutchen KW, Watkins JM, Eberts P, et al. Helical tomotherapy for total lymphoid irradiation. Radiat Med 
2008;26:622-6.

23. Balasz K, Dolla $£$, Giebel S, et al. Total Lymphoid Irradiation: A Comparison of the Dose Distributions of 4 Irradiation Techniques. Int J Radiat Oncol Biol Phys 2014;90:S941-2.

24. Chin C, Hunt S, Robbins R, et al. Long-term follow-up after total lymphoid irradiation in pediatric heart transplant recipients. J Heart Lung Transplant 2002;21:667-73.

25. Salter SP, Salter MM, Kirklin JK, et al. Total lymphoid irradiation in the treatment of early or recurrent heart

doi: $10.21037 /$ tro.2020.04.02

Cite this article as: Lee PH, Wu JK, Wang MC, Lee SH, Kuo $\mathrm{SH}$, Chen YH. A single institution experience of total lymphoid irradiation using helical tomotherapy as part of the conditioning regimen of transplantation for severe aplastic anemia patients. Ther Radiol Oncol 2020;4:6. transplant rejection. Int J Radiat Oncol Biol Phys 1995;33:83-8.

26. Wolden SL, Tate DJ, Hunt SA, et al. Long-term results of total lymphoid irradiation in the treatment of cardiac allograft rejection. Int J Radiat Oncol Biol Phys 1997;39:953-60.

27. Hall EJ, Wuu CS. Radiation-induced second cancers: the impact of 3D-CRT and IMRT. Int J Radiat Oncol Biol Phys 2003;56:83-8. 